\title{
Research
}

\section{Unrecognised bipolar disorder among UK primary care patients prescribed antidepressants:}

\author{
an observational study
}

\begin{abstract}
\section{Background}

Bipolar disorder is not uncommon, is associated with high disability and risk of suicide, often presents with depression, and can go unrecognised.
\end{abstract}

\section{Aim}

To determine the prevalence of unrecognised bipolar disorder among those prescribed antidepressants for depressive or anxiety disorder in UK primary care; whether those with unrecognised bipolar disorder have more severe depression than those who do not; and the accuracy of a screening questionnaire for bipolar disorder, the Mood Disorder Questionnaire (MDQ), in this setting.

\section{Design and setting}

Observational primary care study of patients on the lists of 21 general practices in West Yorkshire aged $16-40$ years and prescribed antidepressant medication.

\section{Method}

Participants were recruited using primary care databases, interviewed using a diagnostic interview, and completed the screening questionnaire and rating scales of symptoms and quality of life.

\section{Results}

The prevalence of unrecognised bipolar disorder was 7.3\%. Adjusting for differences between the sample and a national database gives a prevalence of $10.0 \%$. Those with unrecognised bipolar disorder were younger and had greater lifetime depression. The predictive value of the MDQ was poor.

\section{Conclusion}

Among people aged 16-40 years prescribed antidepressants in primary care for depression or anxiety, there is a substantial proportion with unrecognised bipolar disorder. When seeing patients with depression or anxiety disorder, particularly when they are young or not doing well, clinicians should review the life history for evidence of unrecognised bipolar disorder. Some clinicians might find the MDQ to be a useful supplement to non-standardised questioning.

\section{Keywords}

bipolar disorder; prevalence; primary care; sensitivity and specificity.

\section{INTRODUCTION}

Bipolar disorder affects about $2 \%$ of the population. ${ }^{1}$ It is the sixth leading cause of disability worldwide ${ }^{2}$ and the rate of suicide is over 20 times that of the general population. ${ }^{3}$ When it presents with depression, as it often does, the diagnosis can be difficult. ${ }^{4}$ A history of symptoms indicating mania or hypomania is frequently not recognised as abnormal by the patient and therefore not reported unless specific enquiry is made. Even if such symptoms are reported, they may not be recognised by the clinician. Mild hypomania can be particularly difficult to differentiate from normal mood. Conversely, overdiagnosis results in a medical label that may be permanent and lifelong treatment may be recommended that will benefit few and may harm many.

Studies in primary care outside the UK report that the prevalence of bipolar disorder is between $0.5 \%$ and $4.3 \%$ when structured diagnostic interviews are used, and higher when screening questionnaires are used. These studies do not tell us how much of this bipolar disorder is unrecognised. The studies that have addressed this question are shown in Table 1.7-16 The only UK study reported a median estimate of the prevalence of unrecognised bipolar disorder of $9.6 \%$, much of which was broader bipolar spectrum rather than narrowly defined bipolar disorder. ${ }^{17}$

T Hughes, MD, consultant psychiatrist; K Rolling MRes, research assistant; A Locker, MSc, research assistant, Leeds and York Partnership NHS Foundation Trust, Leeds. A Cardno, PhD, senior lecturer in psychiatry; $\mathbf{R}$ West, DPhil, professor of biostatistics; F Marino-Francis, MA research officer; I Featherstone, PgDip, research assistant; $\mathbf{K}$ McLintock, MA, clinical lecturer in primary care; $\mathbf{A}$ House, DM, professor of liaison psychiatry, Leeds Institute of Health Sciences, University of Leeds, Leeds.
There are unresolved concerns that antidepressant treatment lacks a strong evidence base in bipolar depression, ${ }^{18,19}$ may increase the risk of relapse of mania or hypomania, or worsen the course of bipolar disorder. ${ }^{20-23}$ Most guidelines discourage antidepressant monotherapy in bipolar depression, although some support its use in combination with antimanic drugs for a limited period. 3,24 Therefore, the focus of the present study is on the presence of unrecognised bipolar disorder among those who present with depression or anxiety and are prescribed antidepressant medication.

Although widely used, the National Institute for Health and Care Excellence (NICE) found only two sound studies of the accuracy of the Mood Disorder Questionnaire (MDQ) in this setting. ${ }^{3}$ This was the only instrument evaluated in more than one study, and none were carried out in the UK. As a result, current NICE guidance is unambiguous: do not use questionnaires in primary care to identify bipolar disorder in adults. Because an accurate screening tool for bipolar disorder in primary care could be useful, a study of the MDQ was undertaken as part of the present study.

The aims of this study were to determine:

- What is the prevalence of unrecognised bipolar disorder among people treated with antidepressants in primary care for depressive or anxiety disorder?

\section{Address for correspondence}

Tom Hughes, Leeds and York Partnership NHS Foundation Trust, Linden House, St Mary's Hospital, Greenhill Road, Leeds LS12 3QE, UK.

E-mail: tomhughesanhs.net

Submitted: 7 July 2015; Editor's response: 5 August 2015; final acceptance: 13 October 2015. (c)British Journal of General Practice

This is the full-length article (published online 7 Jan 2016) of an abridged version published in print. Cite this article as: Br J Gen Pract 2016; DOI: 10.3399/bjgp16X683437 


\section{How this fits in}

Bipolar disorder is not uncommon and can often go unrecognised. This study aimed to determine the prevalence of unrecognised bipolar disorder in patients prescribed antidepressants for depressive or anxiety disorder. More rigorous methodology was used than in previous studies, with a similar prevalence of unrecognised bipolar disorder found but in an enhanced sample. When seeing such patients, clinicians should review the life history for evidence of unrecognised bipolar disorder and may find the MDQ to be a useful supplement to nonstandardised questioning.

- Do people with unrecognised bipolar disorder have more severe depression than those without?

- What is the accuracy of the MDQ as a screen for bipolar disorder in UK primary care?

\section{METHOD}

In around $95 \%$ of cases, bipolar disorder begins before the age of 40 years. ${ }^{25} \mathrm{NHS}$ primary care databases were used to identify all patients on the lists of 21 general practices in West Yorkshire who were aged 16-40 years and prescribed antidepressant medication (British National Formulary class 4.3) for depressive or anxiety disorder on the date the relevant primary care database was searched. Exclusions were individuals with known bipolar disorder. schizophrenia, cataplexy, or primary diagnosis of eating disorder; those receiving antidepressants prescribed solely for pain relief, insomnia, or bedwetting; those with dementia; and those unable to speak English. Potential participants were sent a letter by the practice inviting them to speak to a research worker.

Data were collected between December 2010 and March 2012. All participants were asked to complete the $M D Q,{ }^{26}$ which takes about 5 minutes. Thirteen items ask about symptoms of hypo/mania (MDQ1), one whether several symptoms occurred at the same time (MDQ2), and one how much of a problem these caused (MDQ3). The conventional cut-off score is $\geq 7$ on MDQ1, with several symptoms occurring at the same time (MDQ2) and causing at least a moderate problem (MDQ3). The participant put the completed MDQ into an envelope and sealed it so that researchers were blind to $M D Q$ score.

All participants were then interviewed face-to-face using those sections of the Schedules for Clinical Assessment in Neuropsychiatry (SCAN) version $2.1^{27}$ necessary to make a diagnosis of major depression and bipolar disorder. The researchers completing SCAN were trained in its use at a recognised World Health Organization training centre. Demographic data were recorded at the same interview. SCAN allows distinction between DSM-IV28 bipolar I and bipolar I| disorders. Bipolar I is defined by at least one episode of mania lelevated mood that results in marked impairment of functioningl and, usually, at least one of major depression. Bipolar II is defined by at least one episode of hypomania and at least one of major depression. Hypomania requires the same type and number of symptoms as mania, but these must last for a minimum of only 4 days. The symptoms must not cause marked social or occupational impairment, and therefore

\section{Table 1. Studies of the prevalence of unrecognised bipolar disorder in primary care}

\begin{tabular}{|c|c|c|c|c|c|c|}
\hline Study & Country & Method & Prevalence rate $(95 \% \mathrm{Cl})$ & & & \\
\hline Manning et al 1997 & US & Diagnostic interview & 19.0 (12.6 to 27.7$)$ & 1 & + & 1 \\
\hline Sansone et al ${ }^{8} 1998$ & US & Diagnostic interview & 12.8 (5.4 to 27.3 ) & & & \\
\hline Hirschfeld et al 2003 & US & $M D Q$ & $2.0(1.9$ to 2.1$)$ & & & \\
\hline Hirschfeld et al ${ }^{10} 2005$ & US & $M D Q$ & 16.5 (13.7 to 19.9$)$ & & & \\
\hline Das et al" 2005 & US & $M D Q$ & 9.1 (7.5 to 10.9) & & & \\
\hline Awad et al'12 2007 & US & Brief questionnaire & $14.3(12.4$ to 16.4$)$ & & & \\
\hline Kamat et $a l^{13} 2008$ & US & MDQ & 6.9 (5.7 to 8.4) & & $\leftarrow$ & \\
\hline Vermani et al ${ }^{14} 2011$ & Canada & Brief diagnostic interview & 10.7 (8.8 to 13.0 ) & & & $\rightarrow$ \\
\hline Chiu and Chokka ${ }^{15} 2011$ & Canada & $M D Q$ & 27.9 (25.5 to 30.4 ) & & - & \\
\hline Castelo et al' 2012 & Brazil & $M D Q$ & 7.3 (5.6 to 9.4 ) & 0.00 & 0.25 & 0.50 \\
\hline
\end{tabular}

$M D Q=$ Mood Disorder Questionnaire. Those studies recruiting from secondary care are not included unless data are given separately for primary care. Patients with recognised bipolar disorder are removed from the denominator. Where data are supplied, the rate for bipolar I and II only is used, rather than the rate for the broader bipolar spectrum. 
must not require hospitalisation.

CAGE, ${ }^{29}$ a four-question screen for lifetime alcohol misuse, was completed. A positive answer to two questions has high sensitivity and specificity for excessive drinking. ${ }^{30}$ All participants were asked to complete the SF-36 version 2 (SF-36v2) UK English version. ${ }^{31}$ This measures health over the previous 4 weeks. Items are summed and transformed onto a scale from 0 lworst possible health) to 100 (best possible health). Primary and, where appropriate, secondary care records were examined. To calculate the rate of consultation in primary care with a mental health problem, a researcher reviewed the records from each consultation and decided whether that consultation was for a mental health or another problem.

A case vignette was written describing each participant whom the interviewer thought had any suggestion of bipolar disorder. After the interview, data from SCAN and the case vignette were used to complete the Bipolar Affective Disorder Dimension Scale (BADDS). ${ }^{32}$ BADDS has four dimensions, each rated on a 0-100 scale: mania, depression, psychosis, and incongruence of psychotic symptoms. This provides a description of lifetime symptoms that can identify differences in illness severity among those within a single diagnostic category. Inter-rater reliability and face validity are excellent. ${ }^{32}$ The present and worst ever episode of hypomania, and present and representative episode of depression, were scored.

After independent ratings by each researcher, all participants thought to have any suggestion of bipolar disorder were discussed at a consensus meeting generally involving the interviewer and two experienced clinicians. In a small number the vignette, SCAN and BADDS ratings, and diagnostic assignment made by the interviewer were reviewed by these clinicians without the interviewer. Consensus DSM-IV diagnosis and BADDS rating were made.

Data obtained from The Health Improvement Network (THIN), which collects data from over 415 general practices, were used to judge the representativeness of the study sample. The study was powered to give a standard error of the estimate of prevalence of 2.5\%. Analyses were conducted using SPSS (version 22), Medcalc (version 15.2.2), and Stata (version SE 13). Skewed data were log transformed, and data are presented after back-transformation.

To calculate the weighted prevalence of unrecognised bipolar disorder, weights were applied to the sample to give the expected prevalence if the sample contained the same proportions of males and females $>$ and $<30$ years of age as those in the sample from THIN. To examine whether the general practice at which the participant was registered influenced the rate of unrecognised bipolar disorder, a hierarchical model was fitted with participants nested within practices. A generalised linear mixed-model fit with random effects using the Laplace approximation was used. The contribution of independent variables to variation in the dependent variable (the presence or absence of unrecognised bipolar disorderl was examined using logistic regression with independent variables entered using forward likelihood ratio selection. Probability for entry was set at 0.05 and exit at 0.10 .

\section{RESULTS}

\section{Characteristics of participants}

Of 2433 patients identified from primary care data, 92 were excluded by their GP. Of 2341 invited to participate, $236(10.1 \%)$ did so. A further 77 were excluded by their GP before a second invitation was sent. Compared with the 2105 who did not participate, those who participated included a greater proportion of females $(0.77$ versus 0.71 , difference $0.06,95 \% \mathrm{Cl}=0.01$ to 0.12 ), were older (mean age 32.9 years, SD 5.5 versus mean age 31.0 years, SD 6.4, mean difference 1.1 years, $95 \% \mathrm{Cl}=1.0$ to 1.1 ), and the number of days prescription of their most recent antidepressant was greater Imean 430.1 days, SD 588.6 versus mean 384.9 days, SD 536.6, mean difference 1.1 days, $95 \% \mathrm{Cl}=1.1$ to 1.4 ).

Primary and secondary care records showed that three of the 236 participants had received a diagnosis of bipolar disorder that had not been revealed by the electronic database search and were excluded from subsequent analyses. Data from THIN on 12000 adults aged $16-40$ years and prescribed antidepressant medication between 30 November 2010 and 31 January 2012 were extracted in January 2012. Compared with THIN patients, patients in the present study sample were older and a greater proportion were female (Table 2).

The latest clinical diagnosis in primary care was depression in 162 (69.5\%), anxiety disorder in 46 (19.7\%), and mixed depression and anxiety in 24 (10.3\%). One participant did not consent to examination of their primary care record.

Prevalence of unrecognised bipolar disorder and patient characteristics

A diagnosis of bipolar II disorder was made in 17 or $7.3 \%(95 \% \mathrm{Cl}=4.4 \%$ to $11.1 \%)$ of 
Table 2. Comparison of the study sample with THIN sample

\begin{tabular}{lccc} 
Characteristic & Study sample & THIN & Weighting \\
\hline N & 233 & 12000 & \\
Female $18-30$ & $121(51.9 \%)$ & $4085(34.0 \%)$ & 0.655 \\
Female $>30$ & $61(26.2 \%)$ & $3470(28.9 \%)$ & 1.103 \\
Male $18-30$ & $38(16.3 \%)$ & $1986(16.6 \%)$ & 1.018 \\
Male $>30$ & $13(5.6 \%)$ & $2459(20.5 \%)$ & 3.661 \\
\hline
\end{tabular}

Weighting $=$ the weighting of the PAPPA sample required to adjust for differences with the THIN sample.

\section{Table 3. Sample characteristics}

\begin{tabular}{lccc} 
Characteristic & UBD & Not UBD & Difference (95\% Cl) \\
\hline$N(\%)$ & $17(7.3)$ & $216(92.7)$ & \\
Female, $n(\%)$ & $13(77)$ & $169(78)$ & $0.02(-0.13$ to 0.26$)$ \\
Age, mean (SD) & $30.2(6.4)$ & $32.9(5.3)$ & $1.10(0.98 \text { to } 1.23)^{a}$ \\
Married/cohabiting & $5(0.29)$ & $115(0.53)$ & $0.24(-0.01$ to 0.41$)$ \\
White British & $16(0.94)$ & $202(0.94)$ & $0.01(-0.21$ to 0.07$)$ \\
Years of education, mean (SD) & $14.1(1.9)$ & $14.5(2.9)$ & $1.02(0.94 \text { to } 1.10)^{a}$ \\
Age at onset of depression, mean (SD) & $17.5(5.4)$ & $20.5(7.3)$ & $1.24(1.02 \text { to } 1.52)^{a}$ \\
First-degree family history of mood disorder & $5(0.50)$ & $80(0.45)$ & $0.05(-0.22$ to 0.33$)$
\end{tabular}

Proportions are given unless otherwise indicated. SCAN = Schedules for Clinical Assessment in Neuropsychiatry. $U B D=$ unrecognised bipolar disorder. ${ }^{3}$ Back-transformed from transformed data.

\section{Table 4. Association of variables with unrecognised bipolar disorder}

\begin{tabular}{lccc} 
Variable & $\begin{array}{c}\text { UBD, } \\
\text { mean (SD) }\end{array}$ & $\begin{array}{c}\text { Not UBD, } \\
\text { mean (SD) }\end{array}$ & $\begin{array}{c}\text { Mean difference, } \\
\mathbf{9 5 \%} \text { Cl }\end{array}$ \\
\hline Lifetime days AD & $953.1(668.9)$ & $856.1(813.8)$ & $1.5(0.8 \text { to } 2.7)^{\mathrm{a}}$ \\
Lifetime number AD & $2.2(1.6)$ & $2.3(1.5)$ & $0.5(0.7 \text { to } 1.3)^{\mathrm{a}}$ \\
PC appointments & $24.2(24.0)$ & $15.4(10.3)$ & $1.4(0.6 \text { to } 2.0)^{\mathrm{a}}$ \\
Appointment rate & $5.0(4.6)$ & $6.6(11.6)$ & $0.7(0.5 \text { to } 1.9)^{\mathrm{a}}$ \\
BADDS PS depression & $38.1(19.5)$ & $23.6(22.8)$ & $1.4(0.7 \text { to } 2.8)^{\mathrm{a}}$ \\
BADDS LT depression & $59.7(10.7)$ & $50.0(15.4)$ & $1.2(1.0 \text { to } 1.4)^{\mathrm{a}}$ \\
SF36 PHC & $53.6(8.5)$ & $50.6(11.3)$ & $3.1(-2.4$ to 8.5$)$ \\
SF36 MHC & $22.6(11.1)$ & $30.7(13.4)$ & $8.36(1.8$ to 14.9$)$ \\
\hline
\end{tabular}

${ }^{a}$ Back-transformed from transformed data. $A D=$ antidepressant. $B A D D S=$ Bipolar $A$ ffective Disorder Dimension Scale. $L T=$ lifetime. $M H C=$ mental health component. $P C=$ primary care. $P H C=$ physical health component. $P S=$ present state. $U B D=$ unrecognised bipolar disorder. Appointment rate $=$ number of appointments per year for a mental health problem. data (Table 2) from THIN, the prevalence of unrecognised bipolar disorder is $10.0 \%$ $(95 \% \mathrm{Cl}=6.6 \%$ to $14.3 \%)$. The variance fitted to general practice was zero, that is, there was no practice-level contribution.

Four $(24 \%)$ of those with unrecognised bipolar disorder scored $\geq 2$ on CAGE indicating excessive drinking, compared with $60(28 \%)$ of those without unrecognised bipolar disorder. Other characteristics of responders are shown in Table 3. Only two participants with unrecognised bipolar disorder were prescribed mood-stabilising/ antimanic medication: one aripiprazole, the other quetiapine and lithium. The association of unrecognised bipolar disorder with other aspects of mental health is shown in Table 4. The SF-36 mental component score was highly correlated with the BADDS depression present state score $(r=-7.2, P<0.01)$, and total lifetime number of antidepressants prescribed correlated moderately with lifetime days of antidepressant prescription ( $r=0.44, P<0.01)$.

Associations with diagnosis were studied. Age, sex, SF-36 mental component score, BADDS lifetime depression score, total lifetime number of antidepressants prescribed, and consultation rate for mental health problems were entered into a logistic regression model. Higher BADDS lifetime depression score $(\operatorname{ExpB}=1.06$, $95 \% \mathrm{Cl}=1.02$ to $1.10, P=0.008)$ and younger age $(\operatorname{ExpB}=0.90,95 \% \mathrm{Cl}=0.82$ to $0.99, P=0.02$ I were significantly associated with unrecognised bipolar disorder. When the analysis was restricted to those with unrecognised bipolar disorder or DSMIV lifetime major depression, BADDS lifetime depression score $(\operatorname{ExpB}=1.06$, $95 \% \mathrm{Cl}=1.01$ to $1.11, P=0.02$ ) and age ( $\mathrm{ExpB}=0.90,95 \% \mathrm{Cl}=0.82$ to $0.98, P=0.02$ ) remained significantly associated with unrecognised bipolar disorder.

\section{Accuracy of the MDQ}

Individual MDQ1 items left blank $(n=7)$ were scored as zero. Using conventional scoring criteria for MDQ2 and MDQ3, the ROC curve for MDQ1 is shown in Figure 1. Table 5 shows the sensitivity, specificity, and predictive value of the MDQ using conventional scoring. Adjusting the MDQ3 criterion to allow symptoms that caused only a minor problem or no problem at all las might be expected in those with bipolar II) resulted in slightly poorer positive but slightly better negative predictive values.

\section{DISCUSSION}

\section{Summary}

Among people aged $16-40$ years prescribed 


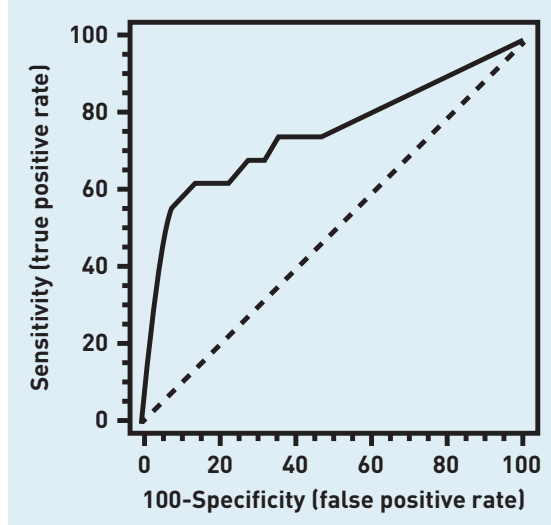

AUC $0.76(95 \% \mathrm{Cl}=0.69$ to 0.81$)$ MDQ score 9

Sensitivity 62.5

Specificity 85.6

Figure 1. Receiver operating characteristic curve for Mood Disorder Questionnaire 1. AUC = area under curve. The point nearest the upper left of the graph is at MDQ1 score $=9$. antidepressant medication in primary care for depression or anxiety there is a substantial proportion with unrecognised bipolar disorder: $7.3 \%$ in the responders of this study and nearly $1 \%$ in the study population ( $n=2341)$, assuming all those with unrecognised bipolar disorder entered the study. Weighting the sample to adjust for possible recruitment bias increases the figure to $10 \%$.

There is a degree of overlap in the treatments available for bipolar disorder and unipolar depression where mood stabilising or antimanic drugs can be used to augment antidepressants. It appeared worthwhile finding out what medication participants were taking because, if those with unrecognised bipolar disorder were getting appropriate treatment, it migh not matter much that bipolar disorder had gone unrecognised. Most of those with unrecognised bipolar disorder were prescribed antidepressants without mood-stabilising/antimanic medication. Compared with those with unipolar major depression, minor depression, or anxiety disorder, those with unrecognised bipolar disorder were younger and reported more severe lifetime depression. The finding of more severe lifetime depression held when comparison was made only with those with current or lifetime DSM-IV major depression. This study shows that unrecognised bipolar disorder, like bipolar depression seen in secondary care, is associated with greater severity of lifetime depression. In this sample all unrecognised bipolar disorder was bipolar $\|$ disorder.
This is not a mild variant of bipolar I, but rather a condition with greater morbidity, comorbidity, and risk of suicide. ${ }^{25,33,34}$

This is the first study to examine the accuracy of the MDQ in UK primary care The large area under the curve shows that the difficulty with the MDQ lies not in the test but in the prevalence of unrecognised bipolar disorder in primary care. This is substantial but far lower than the prevalence in secondary care. Lower prevalence does not affect sensitivity and specificity but, as with any screening test, results in lower positive and higher negative predictive values. The predictive value of the $M D Q$ in this setting was poor. Nevertheless, the $M D Q$ is quick and easy to use and some practitioners may find it useful to augment a clinical interview, bearing in mind that it is not a diagnostic test.

\section{Strengths and limitations}

The strengths of this study include the care taken to exclude those with recognised bipolar disorder from the sample; the large number of practices that participated; the use of a semi-structured standardised diagnostic interview with all participants: and that the reliability of diagnosis was enhanced by consensus meetings with two experienced clinicians. The study has limitations: first, the proportion of those eligible who took part in the study was only $10 \%$. This is in line with other studies, ${ }^{12,13,17}$ but means that the sample may differ systematically from the population. It was possible to make an adjustment for potential recruitment bias by weighting

Table 5. Sensitivity, specificity, and positive and negative predictive values of the MDQ

\begin{tabular}{ccccccccc} 
Cut-off & Sensitivity & $\mathbf{9 5 \%} \mathbf{C l}$ & Specificity & $\mathbf{9 5 \%} \mathbf{C l}$ & $\mathbf{+ P V}$ & $\mathbf{9 5 \%} \mathbf{C l}$ & $\mathbf{- P V}$ & $\mathbf{9 5 \%} \mathbf{C l}$ \\
\hline$\geq 0$ & 100.00 & 79.4 to 100.0 & 0.00 & 0.0 to 1.8 & 7.2 & 4.2 to 11.5 & 100 & 0.99 to 1.00 \\
$\geq 1$ & 100.00 & 79.4 to 100.0 & 0.00 & 0.0 to 1.8 & 7.2 & 4.2 to 11.5 & 100 & 0.99 to 1.00 \\
$\geq 2$ & 75.00 & 47.6 to 92.7 & 53.66 & 46.6 to 60.6 & 11.2 & 5.9 to 18.8 & 96.5 & 91.3 to 99.0 \\
$\geq 3$ & 75.00 & 47.6 to 92.7 & 54.15 & 47.1 to 61.1 & 11.3 & 6.0 to 18.9 & 96.5 & 91.3 to 99.0 \\
$\geq 4$ & 75.00 & 47.6 to 92.7 & 55.12 & 48.0 to 62.1 & 11.5 & 6.1 to 19.3 & 96.6 & 91.5 to 99.1 \\
$\geq 5$ & 75.00 & 47.6 to 92.7 & 59.02 & 52.0 to 65.8 & 12.5 & 6.6 to 20.8 & 96.8 & 92.0 to 99.1 \\
$\geq 6$ & 75.00 & 47.6 to 92.7 & 63.90 & 56.9 to 70.5 & 14.0 & 7.4 to 23.1 & 97.0 & 92.6 to 99.2 \\
$\geq 7$ & 68.75 & 41.3 to 89.0 & 67.80 & 60.9 to 74.1 & 14.3 & 7.4 to 24.1 & 96.5 & 92.1 to 98.9 \\
$\geq 8$ & 68.75 & 41.3 to 89.0 & 72.20 & 65.5 to 78.2 & 16.2 & 8.4 to 27.1 & 96.7 & 92.5 to 98.9 \\
$\geq 9$ & 62.50 & 35.4 to 84.8 & 77.07 & 70.7 to 82.6 & 17.5 & 8.7 to 29.9 & 96.3 & 92.2 to 98.6 \\
$\geq 10$ & 62.50 & 35.4 to 84.8 & 85.85 & 80.3 to 90.3 & 25.6 & 13.0 to 42.1 & 96.7 & 93.0 to 98.8 \\
$\geq 11$ & 56.25 & 29.9 to 80.2 & 92.20 & 87.6 to 95.5 & 36.0 & 18.0 to 57.5 & 96.4 & 92.8 to 98.6 \\
$\geq 12$ & 37.50 & 15.2 to 64.6 & 96.10 & 92.5 to 98.3 & 42.9 & 17.7 to 71.1 & 95.2 & 91.3 to 97.7 \\
$=13$ & 25.00 & 7.3 to 52.4 & 97.56 & 94.4 to 99.2 & 44.4 & 13.7 to 78.8 & 94.3 & 90.3 to 97.0 \\
\hline
\end{tabular}

$M D Q=$ Mood Disorder Questionnaire. $+P V=$ positive predictive value. $-P V=$ negative predictive value. 
the main finding in accordance with the large dataset from THIN. Nevertheless, the result should be viewed with caution. For example, participants may have been more likely to be those who doubted their diagnosis or doubted the effectiveness of antidepressants. This could result in an overestimate of prevalence. Second, patients are more likely to fail to remember past episodes of hypomania than to report episodes that have not occurred. Third, collateral history was rarely obtained from an informant who may have remembered past episodes of hypomania. The likely direction of the last two potential biases would be to underestimate the prevalence of unrecognised bipolar disorder. Fourth, the determination of whether primary care appointments were for a mental health or other problem involved some subjective judgement.

\section{Comparison with existing literature}

In this sample the prevalence of unrecognised bipolar disorder was enhanced by inclusion of those taking antidepressants and use of an upper age limit. Studies outside the UK have almost always used screening questionnaires. Such questionnaires will record many more false positives than false negatives and will necessarily have included bipolar spectrum conditions in the rate. The only UK primary care study included a more loosely defined bipolar spectrum in the prevalence estimate. The present study used more rigorous methodology and found a prevalence similar to other studies, but in an enhanced sample. The finding that these patients are younger supports the findings of other studies, ${ }^{9,12}$ and may reflect the greater incidence of bipolar disorder at a younger age. ${ }^{35,36}$

\section{Implications for research and practice}

Primary care clinicians should review life histories for evidence of unrecognised bipolar disorder, particularly bipolar II disorder, when seeing patients with depression or anxiety disorder, particularly younger patients and those who are not doing well.

Could screening with the MDQ in routine practice be a means of supporting this general aim? Using the conventional cut-off score on $M D Q$ of $\geq 7$, even in the selected population studied here, only around 14\% of high scorers will have bipolar disorder, and somewhere between $1 \%$ and $8 \%$ of those currently with unrecognised bipolar disorder will be missed. The present data give support from a UK sample to guidance that advocates enquiry about a history of symptoms of hypomania in all those presenting with depression, ${ }^{3}$ and suggest this should also apply to those presenting with anxiety. Given the relative rarity of the condition under study it is unlikely that routine case finding using the MDQ will find much favour with GPs, but it may prove useful as a supplement to non-standardised questioning for those who are considering referral for specialist assessment in the mental health service.

The present data are consistent with the view that antidepressants alone, without concurrent mood-stabilising or antimanic medication, are less effective in bipolar ॥ depression than in unipolar depression. Better data are required on which treatments are effective for this group, from samples of people with bipolar II rather than bipolar I disorder or unipolar depression. It would be useful also to find out whether the predictive value of the MDQ can be improved by enquiring about duration of symptoms.
Approval was obtained from Leeds East Research Ethics Committee (09/ H1306/118). Participants gave informed consent before taking part.

\section{Provenance}

Freely submitted; externally peer reviewed.

\section{Competing interests}

The authors have declared no competing interests.

\section{Acknowledgements}

We thank Lucas Coulson, and the staff and patients from participating general practices including the One Medicare group. We also thank The Health Improvement Network for providing data.

\section{Discuss this article}

Contribute and read comments about this article: bjgp.org/letters 


\section{REFERENCES}

1. Merikangas KRP, Akiskal HSMD, Angst JMD, et al. Lifetime and 12-month prevalence of bipolar spectrum disorder in the National Comorbidity Survey replication. Arch Gen Psychiatry 2007; 64(5): 543-552.

2. Murray CJL, Lopez AD, eds. The global burden of disease: a comprehensive assessment of mortality and disability from diseases, injuries, and risk factors in 1990 and projected to 2020. Cambridge, MA: Harvard School of Public Health on behalf of the World Health Organization and the World Bank, 1996.

3. National Institute for Health and Care Excellence. Bipolar disorder: the assessment and management of bipolar disorder in adults, children and young people in primary and secondary care. CG185. 2014. https://www.nice.org.uk/ guidance/cg185 (accessed 19 Nov 2015).

4. Angst J. Do many patients with depression suffer from bipolar disorder? Can J Psychiatry 2006; 51(1): 3-5.

5. Moynihan R, Doust J, Henry D. Preventing overdiagnosis: how to stop harming the healthy. BMJ 2012; 344: e3502.

6. Cerimele JM, Chwastiak LA, Dodson S, Katon WJ. The prevalence of bipolar disorder in general primary care samples: a systematic review. Gen Hosp Psychiatry 2014; 36(1): 19-25.

7. Manning JS, Haykal RF, Connor PD, Akiskal HS. On the nature of depressive and anxious states in a family practice setting: the high prevalence of bipolar II and related disorders in a cohort followed longitudinally. Compr Psychiatry 1997; 38(2): 102-108

8. Sansone RA, Wiederman MW, Sansone LA, Touchet B. An investigation of primary care patients receiving extended treatment with selective serotonin reuptake inhibitors. Am J Manag Care 1998; 4(10): 1397-1402.

9. Hirschfeld RMA, Calabrese JR, Weissman MM, et al. Screening for bipolar disorder in the community. J Clin Psychiatry 2003; 64(1): 53-59.

10. Hirschfeld RMA, Cass AR, Holt DCL, Carlson CA. Screening for bipolar disorder in patients treated for depression in a family medicine clinic. J Am Board Fam Pract 2005; 18(4): 233-239

11. Das AK, Olfson M, Gameroff MJ, et al. Screening for bipolar disorder in a primary care practice. JAMA 2005; 293(8): 956-963.

12. Awad AG, Rajagopalan K, Bolge SC, et al. Quality of life among bipolar disorder patients misdiagnosed with major depressive disorder. Prim Care Companion $J$ Clin Psychiatry 2007; 9(3): 195-202.

13. Kamat SA, Rajagopalan K, Pethick N, et al. Prevalence and humanistic impact of potential misdiagnosis of bipolar disorder among patients with major depressive disorder in a commercially insured population. J Manag Care Pharm 2008; 14(7): 632-642

14. Vermani M, Marcus M, Katzman MA. Rates of detection of mood and anxiety disorders in primary care: a descriptive, cross-sectional study. Prim Care Companion CNS Disord 2011; 13(2): e1-e10.

15. Chiu JF, Chokka PR. Prevalence of Bipolar Disorder symptoms in Primary Care (ProBiD-PC): a Canadian study. Can Fam Physician 2011; 57(2): e58-e67.

16. Castelo MS, Hyphantis TN, Macêdo DS, et al. Screening for bipolar disorder in the primary care: a Brazilian survey. J Affect Disord 2012; 143(1-3): 118-124.

17. Smith DJ, Griffiths E, Kelly M, et al. Unrecognised bipolar disorder in primary care patients with depression. Br J Psychiatry 2011; 199(1): 49-56.
18. Goodwin GM. Evidence-based guidelines for treating bipolar disorder: revised second edition - recommendations from the British Association for Psychopharmacology. J Psychopharmacol 2009; 23(4): 346-388.

19. Taylor DM, Cornelius V, Smith L, et al. Comparative efficacy and acceptability of drug treatments for bipolar depression: a multiple-treatments meta-analysis. Acta Psychiatr Scand 2014; 130(6): 452-469.

20. Gijsman HJ, Geddes JR, Rendell JM, et al. Antidepressants for bipolar depression: a systematic review of randomized, controlled trials. Am J Psychiatry 2004; 161(9): 1537-1547.

21. Sidor MM, Macqueen GM. Antidepressants for the acute treatment of bipolar depression: a systematic review and meta-analysis. J Clin Psychiatry 2011; 72(2): 156-167.

22. Ghaemi S, Hsu D, Soldani F, et al. Antidepressants in bipolar disorder: the case for caution. Bipolar Disord 2003; 5(6): 421-433.

23. Licht RW, Gijsman H, Nolen WA, Angst J. Are antidepressants safe in the treatment of bipolar depression? A critical evaluation of their potential risk to induce switch into mania or cycle acceleration. Acta Psychiatr Scand 2008; 118(5): 337-346

24. Nivoli AM, Colom F, Murru A, et al. New treatment guidelines for acute bipolar depression: a systematic review. J Affect Disord 2011; 129(1-3): 14-26.

25. Goodwin FK, Jamison KR. Manic-depressive illness: bipolar disorders and recurrent depression. 2nd edn. New York, NY: Oxford University Press, 2007

26. Hirschfeld RM, Williams JB, Spitzer RL, et al. Development and validation o a screening instrument for bipolar spectrum disorder: the Mood Disorder Questionnaire. Am J Psychiatry 2000; 157(11): 1873-1875.

27. Wing JK, Babor T, Brugha T, et al. SCAN: Schedules for Clinical Assessment in Neuropsychiatry. Arch Gen Psychiatry 1990; 47(6): 589-593.

28. American Psychiatric Association. Diagnostic and statistical manual of mental disorders DSM-IV-TR. 4th edn, text revision edn. Washington, DC: American Psychiatric Association, 2000.

29. Mayfield D, McLeod G, Hall P. The CAGE questionnaire: validation of a new alcoholism screening instrument. Am J Psychiatry 1974; 131(10): 1121-1123.

30. Bernadt MW, Mumford J, Taylor C, et al. Comparison of questionnaire and laboratory tests in the detection of excessive drinking and alcoholism. Lancet 1982; 319(8267): 325-328.

31. Ware JE Jr. Sherbourne CD. The MOS 36-item short-form health survey (SF-36): I. Conceptual framework and item selection. Med Care 1992; 30(6): 473-483

32. Craddock N, Jones I, Kirov G, Jones L. The Bipolar Affective Disorder Dimension Scale (BADDS) - a dimensional scale for rating lifetime psychopathology in bipolar spectrum disorders. BMC Psychiatry 2004; 4: 19.

33. Angst J, Gamma A, Bowden CL, et al. Evidence-based definitions of bipolar-I and bipolar-II disorders among 5,635 patients with major depressive episodes in the Bridge Study: validity and comorbidity. Eur Arch Psychiatry Clin Neurosci 2013; 263(8): 663-673.

34. Saunders KEA, Goodwin GM. The course of bipolar disorder. Adv Psychiatr Treat 2010; 16(5): 318-328.

35. Larsson S, Lorentzen S, Mork E, et al. Age at onset of bipolar disorder in a Norwegian catchment area sample. J Affect Disord 2010; 124(1-2): 174-177.

36. Baldessarini RJ, Bolzani L, Cruz N, et al. Onset-age of bipolar disorders at six international sites. J Affect Disord 2010: 121(1-2): 143-146. 